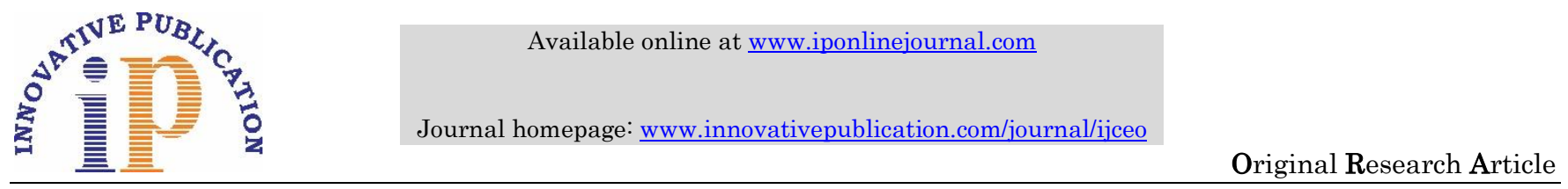

\title{
Comparison of accuracy of haigis and SRK II formula for intraocular lens power calculation in eyes with short axial length
}

\author{
Mohan Kumar $\mathbf{H}^{1}$, Sangeetha $\mathbf{T}^{2}$, Kanthamani ${ }^{3}$, Nithiya Joe Babu ${ }^{4 *}$ \\ ${ }^{1,3}$ Professor, ${ }^{2}$ Associate Professor, ${ }^{4}$ Junior Resident, Dept. of Ophthalmology, Sri Devraj Urs Medical College, Kolar, Karnataka, India
}

\section{Article Info}

Received: $12^{\text {th }}$ March, 2019

Accepted: $23^{\text {rd }}$ April, 2019

Published Online: $9^{\text {th }}$ September, 2019

Keywords: Formula, Haigis, IOL power calculations, SRK II, Short axial length.

\begin{abstract}
Introduction: This study aimed to measure the postoperative residual spherical error and to find the correlation between the axial length and anterior chamber depth measured preoperatively with the postoperative refractive status.

Materials and Methods: A total of 174 patients of the age group 40-70 years axial length $\leq 22$ $\mathrm{mm}$ undergoing cataract surgery were included in our prospective study in which the accuracy of SRK II was compared with Haigis formula. The participants were divided into group A and B by simple randomization technique. The subjects under group A were implanted with IOL calculated using Haigis formula and those under group B with SRK II formula. They were evaluated for visual acuity and residual refractive error. The degree of emmetropia of the participants was analysed using above data.

Results: A total of 174 eyes were included in our study of which 64 (36.7\%) were males and $110(63.2 \%)$ were females. The mean axial length was $21.47 \mathrm{~mm}$ and the mean anterior chamber depth was $3.17 \mathrm{~mm}$. Significant differences were found between the spherical error calculated by SRK II formula and Haigis formula. The mean spherical error in group A with Haigis formula was - 0.36 (range 0 D to -1.5) meanwhile group B with SRK II formula had a mean spherical error of +0.55 (range 0 to $+2 \mathrm{D}$ ). The postoperative refraction of patients implanted with IOLs calculated using Haigis formula was closer to emmetropia.

Conclusion: It was found that in patients with short axial length, Haigis formula gave an accurate prediction of the IOL power compared to SRK II formula. Using Haigis formula led to a mild over correction leading to a slightly more myopic outcome while SRK II formula led to a mild under correction leading to a mild hypermetropic outcome.
\end{abstract}

\section{Introduction}

Senile cataract is a leading cause of blindness in India and other developing countries accounting for about three quarters of the blindness. ${ }^{1}$ The cornerstone of the management of cataract being cataract extraction with intraocular lens implantation (IOL). Cataract has evolved from a mere rehabilitation to a refractive procedure. This is due to precise preoperative biometry, intraoperative lens (IOL) power calculations and improved surgical techniques.

Before the emergence of intraocular lens, patients had to wear thick glasses following cataract surgery. Today several types of IOLs are available for natural lens replacement, helping millions to enjoy clear vision after cataract surgery. IOLs were approved by FDA since early 1980s, and since then modern advances in IOL technology have significantly improved the clarity of vision they can provide. However advanced the IOL maybe, the key to obtaining good refractive outcome after cataract surgery lies in accurate lens power calculation. ${ }^{2}$
A wide range of formulae using different variables like corneal curvature, axial length (AL) and anterior chamber depth (ACD) can be used to calculate the IOL power. ${ }^{3}$

Among the variables, disparity in the axial length, both long and short eyes, can lead to wrong IOL power calculation if appropriate formulae are not employed. ${ }^{4}$ Sanders-Retzlaff-Kraff II (SRK II) formula which is widely practiced may not be useful in IOL power calculation in long and short eyes. ${ }^{5}$

In eyes with short $\mathrm{AL}$, Haigis formula is useful for calculating IOL power length and to estimate the postoperative effective lens position (ELP) by using preoperative anterior chamber depth and axial length. ${ }^{3,6,7}$ If SRK II formula is used in eyes with short AL, it might lead to poor visual outcome, which can be minimized by adopting Haigis formula in such situations. This study aimed to evaluate the accuracy of Haigis formula and SRK II formula in eyes with short AL and to find the correlation between the axial length and anterior chamber depth measured preoperatively with the postoperative refractive status.

\footnotetext{
*Corresponding Author: Nithiya Joe Babu, Junior Resident, Dept. of Ophthalmology, Sri Devraj Urs Medical College, Kolar, Karnataka, India

Email: njoybabu@gmail.com

http://doi.org/10.18231/j.ijceo.2019.090
} 


\section{Materials and Methods Study Population}

A total of 174 eyes which fulfilled the inclusion criteria were selected and allotted into 2 groups (87 in each group) by simple randomization technique for this prospective study which was conducted in Ophthalmology outpatient department at R.L. Jalappa hospital and research centre, Tamaka, Kolar attached to Sri Devaraj Urs medical college between December 2017 and December 2018. Our study consisted of patients of the age group 40-70 years with $\mathrm{AL} \leq$ $22 \mathrm{~mm}$ posted for cataract surgery. Those who had corneal disorders like corneal opacity, degenerations and dystrophies, retinal diseases like diabetic retinopathy, macular degeneration, history of previous ocular surgeries, pseudo exfoliation, subluxated/ traumatic cataract, severe dry eye, glaucoma and uncontrolled diabetes were excluded from our study.

\section{Method of Collection of Data}

All patients underwent similar protocol for standard cataract evaluation, which included recording of visual acuity, intraocular pressure, slit lamp examination and fundus evaluation.

Routine blood investigations like complete blood count (CBC), random blood sugar (RBS), HIV, HBsAg, serum urea creatinine was done.

Preoperative keratometry was measured using a standard calibrated manual Bausch and Lomb keratometer, axial length, and intraocular lens power calculation by SRK II and Haigis formulae by A- Scan (Appasamy Associates).

All patients received xylocaine test dose, oral tab Ciprofloxacin $500 \mathrm{mg}$ BD and Ciprofloxacin $0.3 \%$ eye drops hourly one day before the surgery.

Preoperatively pupils were dilated using topicamide with phenylephrine $0.5 \%$ or $1 \%$ eye drops along with flurbiprofen $0.03 \%$ drops.

The participants were divided into group A and B by simple randomization technique.

Intraoperatively, patients were subjected to manual small incision cataract surgery with posterior chamber IOL implantation (in the bag) by a single surgeon.

The subjects under group A were implanted with IOL calculated using Haigis formula:

Haigis formula $=\mathrm{a}_{0}+\mathrm{a}_{1} \mathrm{ACD}+\mathrm{a}_{2} \mathrm{AL}$

$\mathrm{ACD}=$ anterior chamber depth in $\mathrm{mm}$

$\mathrm{AL}=$ axial length in $\mathrm{mm}$

$a_{0}, a_{1}, a_{2}$ constants in Haigis formula were set by optimizing a set of surgeon - IOL specific outcomes for a wide range of $\mathrm{AL}$ and $\mathrm{ACD}$

The subjects under group B were implanted with IOL calculated using SRK II formula:

SRK II formula $=\mathrm{A}-2.5 \mathrm{~L}-0.9 \mathrm{~K}$

$\mathrm{P}=\mathrm{IOL}$ power

$\mathrm{A}=$ constant specific for each lens

$\mathrm{AL}=$ axial length in $\mathrm{mm}$
$\mathrm{K}=$ average keratometry in dioptres

If $\mathrm{L}$ is < 20mm: $\mathrm{A}+3$

If $\mathrm{L}$ is $20-20.99 \mathrm{~mm}: \mathrm{A}+2$

If $\mathrm{L}$ is 21-21.99: $\mathrm{A}+1$

If $\mathrm{L}$ is $22-24.5$ : $\mathrm{A}$

If $\mathrm{L}$ is >24.5: $\mathrm{A}-0.5$

Postoperative medications include an antibiotic steroid eye drops that was used for 6 weeks in a tapering dose. All participants were followed up from $1^{\text {st }}$ day, $1^{\text {st }}$ week, $4^{\text {th }}$ week and $6^{\text {th }}$ week and at each visit patient was evaluated for visual acuity and residual refractive error.

The degree of emmetropia of the participants was analysed using above data. The accuracy of the intraocular lens power formulae was arrived by measuring the objective refractive error by autorefractometer postoperatively (allowance factor for spherical error being \pm 0.25 ).

\section{Statistical Analysis}

Collected data will be coded into an Excel spreadsheet with all the quantitative measures expressed by mean and standard deviation (SD) with confidence interval and quantitative data by proportions.

Student $\mathrm{t}$ test / Mann Whitney $\mathrm{U}$ test was used to compare difference of means. Chi square test for testing difference in proportion. Sample linear regression was used to find out the difference between anterior chamber depth and refractive error.

$\mathrm{p}$ value $\leq 0.05$ was interpreted as being statistically significant.

\section{Results}

A total of 174 eyes of 174 subjects were included in our study of which 64(36.7\%) were males and 110(63.2\%) were females. The number of females were more in both the groups (Table 1).

Table 1: Sex distribution

\begin{tabular}{|c|c|c|c|}
\hline & Male & Female & Total \\
\hline Group A & $36(41.3 \%)$ & $51(58.62 \%)$ & 87 \\
\hline Group B & $28(32.3 \%)$ & $59(67.8 \%)$ & 87 \\
\hline Total & $64(36.7 \%)$ & $110(63.2 \%)$ & 174 \\
\hline
\end{tabular}

49(56.3\%) subjects from group A and 23(26.4\%) from group B achieved 6/6 vision. 32(36.8\%) and 44(50.6\%) subjects gained an uncorrected visual acuity (UCVA) of 6/9 from group A and B respectively. 6(6.8\%) subjects from group A and 18 (20.6\%) subjects from group B achieved UCVA of 6/12. All subjects from group A gained UCVA of $\geq 6 / 12$ while 2 patients from group B had UCVA worse than $6 / 12$.

From group A $81(93.1 \%)$ subjects gained BCVA of $6 / 6$ and $6(6.8 \%)$ gained BCVA of $6 / 9$ while from group B 80 (92\%) gained BCVA of $6 / 6$ and $7(8 \%)$ gained BCVA of $6 / 9$. Thus, all patients achieved a best corrected visual acuity of $6 / 6$ except 13 subjects. 
Table 2: Clinical characteristics of study participants, and their residual spherical power

\begin{tabular}{|c|c|c|c|c|c|c|}
\hline \multicolumn{2}{|c|}{ Group } & $\begin{array}{c}\text { AGE } \\
\text { (in years) }\end{array}$ & AL (in mm) & $\begin{array}{c}\text { ACD } \\
\text { in mm) }\end{array}$ & $\begin{array}{c}\text { IOL Power } \\
\text { (in Diopters) }\end{array}$ & $\begin{array}{c}\text { Residual Spherical } \\
\text { Power (in Diopters) }\end{array}$ \\
\hline \multirow{4}{*}{ Haigis } & Mean & 65.07 & 21.427126 & 3.154598 & 28.920 & -.3592 \\
\cline { 2 - 7 } & $\mathrm{N}$ & 87 & 87 & 87 & 87 & 87 \\
\cline { 2 - 7 } & SD & 6.511 & .5579675 & .2021213 & 3.2989 & .28960 \\
\cline { 2 - 7 } & Minimum & 50 & 20.0000 & 2.4200 & 23.0 & -1.50 \\
\cline { 2 - 7 } & Maximum & 82 & 22.0000 & 3.6700 & 36.0 & .00 \\
\hline SRK II & Mean & 65.43 & 21.520345 & 3.196667 & 26.318 & .5374 \\
\cline { 2 - 7 } & $\mathrm{N}$ & 87 & 87 & 87 & 87 & .35258 \\
\cline { 2 - 7 } & SD & 8.835 & .5261874 & .1667310 & 2.6575 & .00 \\
\cline { 2 - 7 } & Minimum & 44 & 20.0000 & 2.8600 & 21.0 & 2.00 \\
\cline { 2 - 7 } & Maximum & 90 & 22.0000 & 3.4400 & 31.0 & \\
\hline \multirow{2}{*}{$\begin{array}{l}\text { N= Number of participants } \\
\text { SD=standard deviation }\end{array}$} & & & & \\
\hline
\end{tabular}

The 174 eyes analysed had a mean AL of $21.47 \mathrm{~mm}$ (range 20-22mm) and a mean ACD of $3.17 \mathrm{~mm}$ (range 2.42-3.67mm). However, the mean AL of group A was $21.42 \mathrm{~mm}$ (range 20-22mm) and the ACD was $3.15 \mathrm{~mm}$ (range 2.42-3.67mm). In group B the mean axial length was $21.52 \mathrm{~mm}$ (range $20-22 \mathrm{~mm}$ ) and the anterior chamber depth was $3.19 \mathrm{~mm}$ (range 2.86$3.44 \mathrm{~mm}$ ) (Table 2).

For an axial length between 21 and $22 \mathrm{~mm}$ the anterior chamber depth was 2.86-3. 42mm.The ACD was 2.96-3.27mm when the AL was between $20-21 \mathrm{~mm}$ and $2.88-3.04 \mathrm{~mm}$ when it was $<20 \mathrm{~mm}$.

Table 3: Spherical error obtained by autorefractometer

\begin{tabular}{|c|c|c|c|c|c|c|c|c|}
\hline SE & Emmetropia & $\pm 0.25 \mathrm{D}$ & $\pm 0.5 \mathrm{D}$ & $\pm 0.75 \mathrm{D}$ & $\pm 1 \mathrm{D}$ & $\pm 1.5 \mathrm{D}$ & $\pm 2 \mathrm{D}$ & $< \pm 2 \mathrm{D}$ \\
\hline Group A & 17 & 34 & 27 & 4 & 5 & - & - & - \\
\hline Group B & 3 & 16 & 50 & 5 & 10 & 1 & 2 & - \\
\hline $\begin{array}{l}\text { SE- Spheri } \\
\text { Group A- i } \\
\text { Group B-i }\end{array}$ & IOL & & & & & & & \\
\hline
\end{tabular}

With Haigis formula 17 eyes (19.5\%) and with SRK II formula 3 eyes (3.5\%) achieved emmetropia. 34 eyes (39\%) in group A and 16 eyes (i.e., 18.4\%) in group B possessed a residual spherical error of \pm 0.25 . In group A $27(31 \%)$ had a residual spherical error of \pm 0.5 while $50(57.5 \%)$ eyes had a residual spherical error of \pm 0.5 in group B. 4 eyes (4.5\%) in group A and 5 eyes $(5.75 \%)$ in group B had a residual spherical error of \pm 0.75 . Only 5 eyes $(5.75 \%)$ had a residual spherical error of $1 \mathrm{D}$ while 10 eyes $(11.5 \%)$ in group B had the same (Table 3 ).

Table 4.

\begin{tabular}{|c|c|c|c|c|c|c|c|}
\hline & Group & $\mathbf{N}$ & Mean & Std. Deviation & Std. Error Mean & t Value & P-Value \\
\hline \multirow{2}{*}{$\begin{array}{c}\text { Residual Spherical } \\
\text { Power }\end{array}$} & Haigis & 87 & -.3592 & .28960 & .03105 & 18.33 & $<0.001^{* *}$ \\
\cline { 2 - 8 } & SRK II & 87 & .5374 & .35258 & .03780 & & \\
\hline \multirow{2}{*}{ Age } & Haigis & 87 & 65.07 & 6.511 & .698 & 0.303 & 0.762 \\
\cline { 2 - 8 } & SRK II & 87 & 65.43 & 8.835 & .947 & & \\
\hline \multirow{2}{*}{ AL } & Haigis & 87 & 21.427126 & .5579675 & .0598204 & 1.134 & 0.258 \\
\cline { 2 - 7 } & SRK II & 87 & 21.520345 & .5261874 & .0564132 & & \\
\hline \multirow{2}{*}{ ACD } & Haigis & 87 & 3.154598 & .2021213 & .0216697 & \multirow{2}{*}{1.498} & \multirow{2}{*}{0.136} \\
\cline { 2 - 7 } & SRK II & 87 & 3.196667 & .1667310 & .0178754 & & \\
\hline \multirow{2}{*}{ IOL Power } & Haigis & 87 & 28.920 & 3.2989 & .3537 & 5.727 & $<0.001 *$ \\
\cline { 2 - 7 } & SRK II & 87 & 26.318 & 2.6575 & .2849 & & \\
\hline
\end{tabular}

The mean spherical error in group A was - 0.36 (range $0 \mathrm{D}$ to -1.5 ) meanwhile group B had a mean spherical error of +0.55 (range 0 to $+2 \mathrm{D}$ ). The mean differences observed in residual spherical power between Haigis method and SRK II method is statistically significant, $(\mathrm{P}<0.001)$, The mean differences observed in IOL power between Haigis method and SRK II method is statistically significant, $(\mathrm{P}<0.001)$ (Table 4). 
Table 5: Correlation between the axial length and anterior chamber depth measured preoperatively with the postoperative refractive status

\begin{tabular}{|l|c|c|c|c|c|l|}
\hline & \multicolumn{2}{|c|}{ Haigis } & & \multicolumn{2}{c|}{ SRK II } & \\
\hline & $\begin{array}{l}\text { Correlation } \\
\text { Coefficient }\end{array}$ & P-Value & & $\begin{array}{l}\text { Correlation } \\
\text { Coefficient }\end{array}$ & P-Value & \\
\hline $\begin{array}{l}\text { Axial Length and } \\
\text { residual spherical } \\
\text { power }\end{array}$ & -0.214 & $0.047^{*}$ & $\begin{array}{l}\text { Correlation is } \\
\text { negative and } \\
\text { significant }\end{array}$ & -0.279 & $0.009^{*}$ & $\begin{array}{l}\text { Correlation is negative } \\
\text { and significant }\end{array}$ \\
\hline $\begin{array}{l}\text { ACD and residual } \\
\text { spherical power }\end{array}$ & 0.029 & 0.798 & & -0.174 & 0.107 & \\
\hline $\begin{array}{l}\text { IOL power and residual } \\
\text { spherical power }\end{array}$ & 0.157 & 0.148 & & 0.256 & $0.017^{*}$ & $\begin{array}{l}\text { Correlation is Positive } \\
\text { and significant }\end{array}$ \\
\hline
\end{tabular}

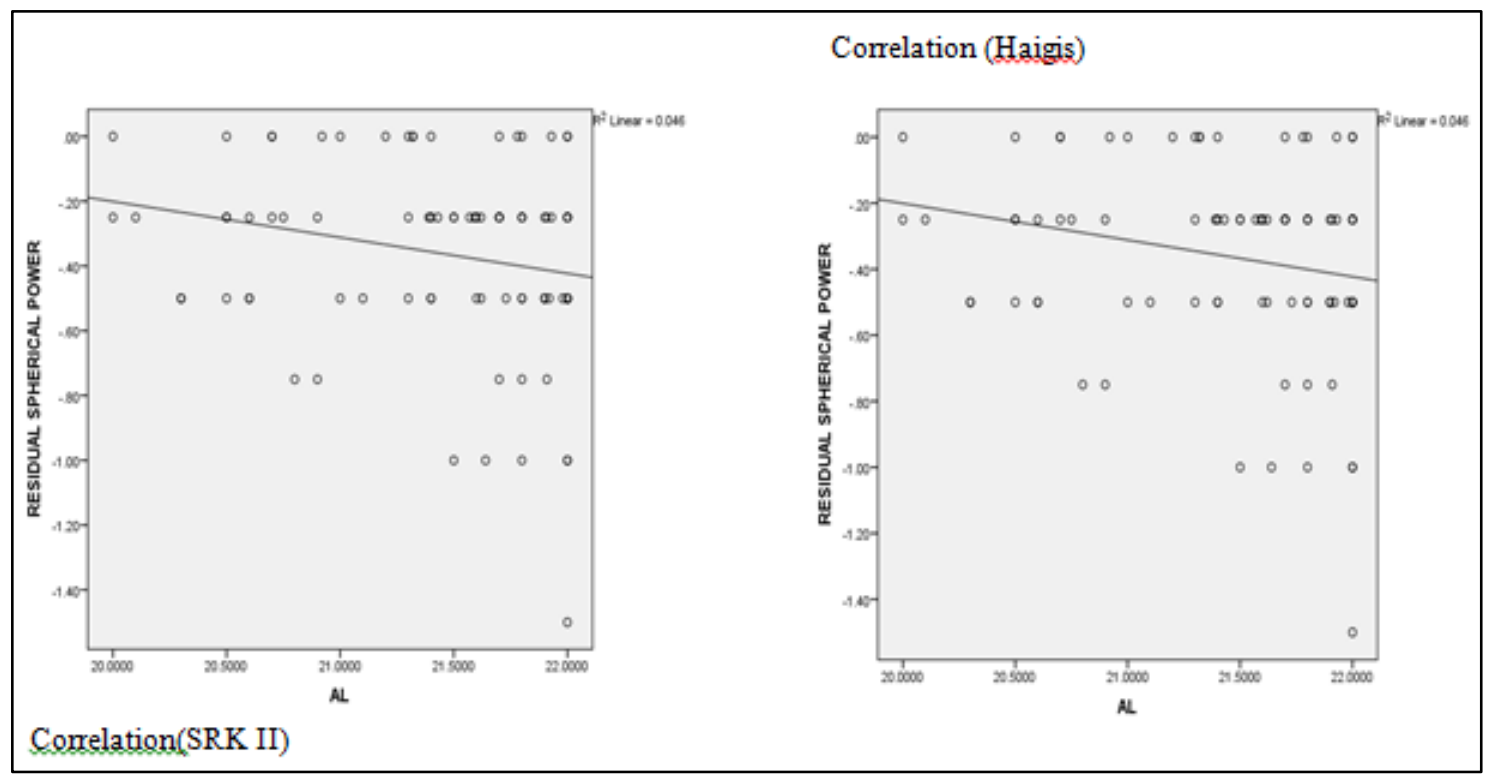

Fig. 2: Comparison of axial length with residual spherical power in Haigis and SRK II formulae.

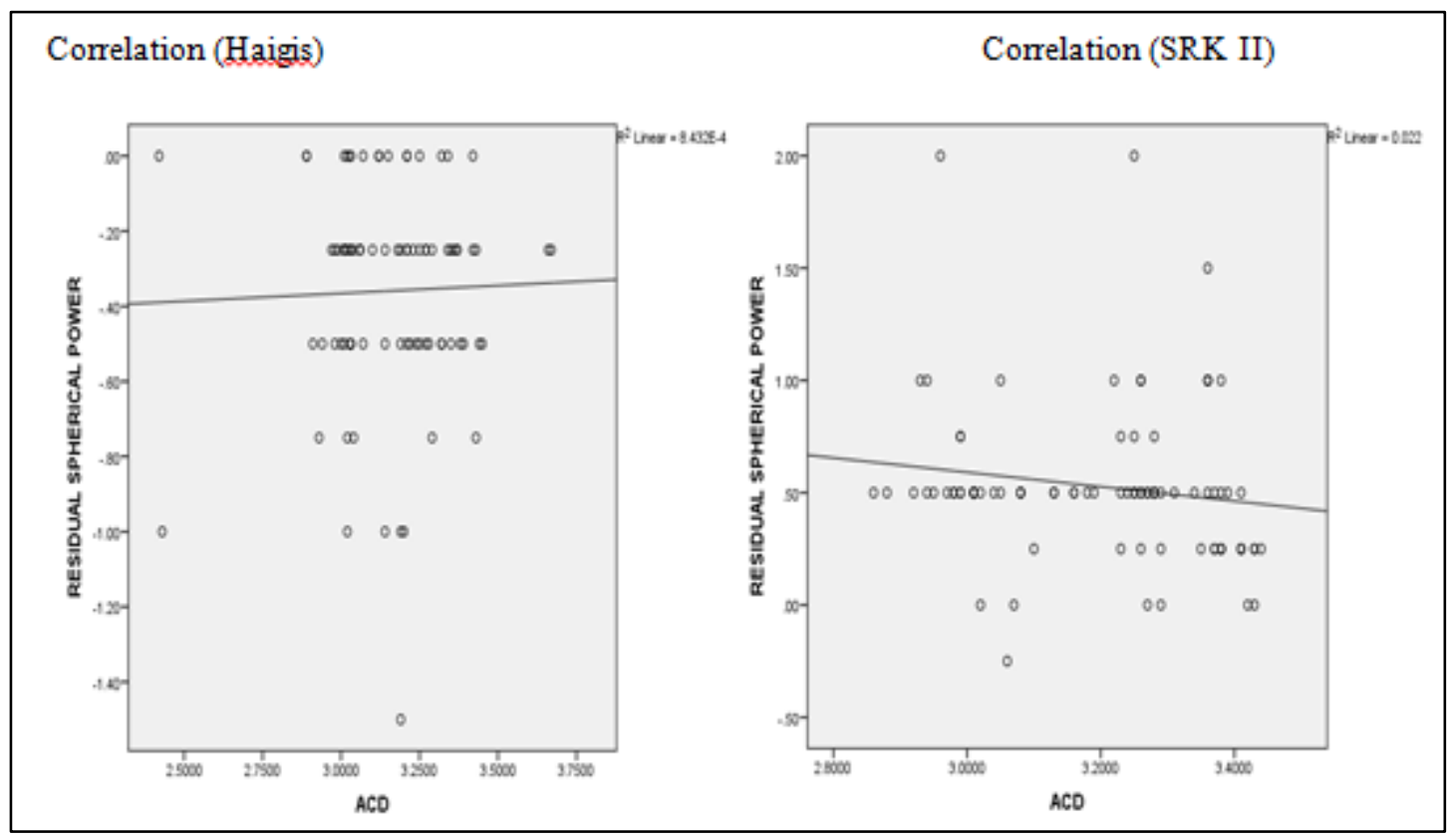

Fig. 3: Comparison of anterior chamber depth with residual spherical power in Haigis and SRK II formulae. 


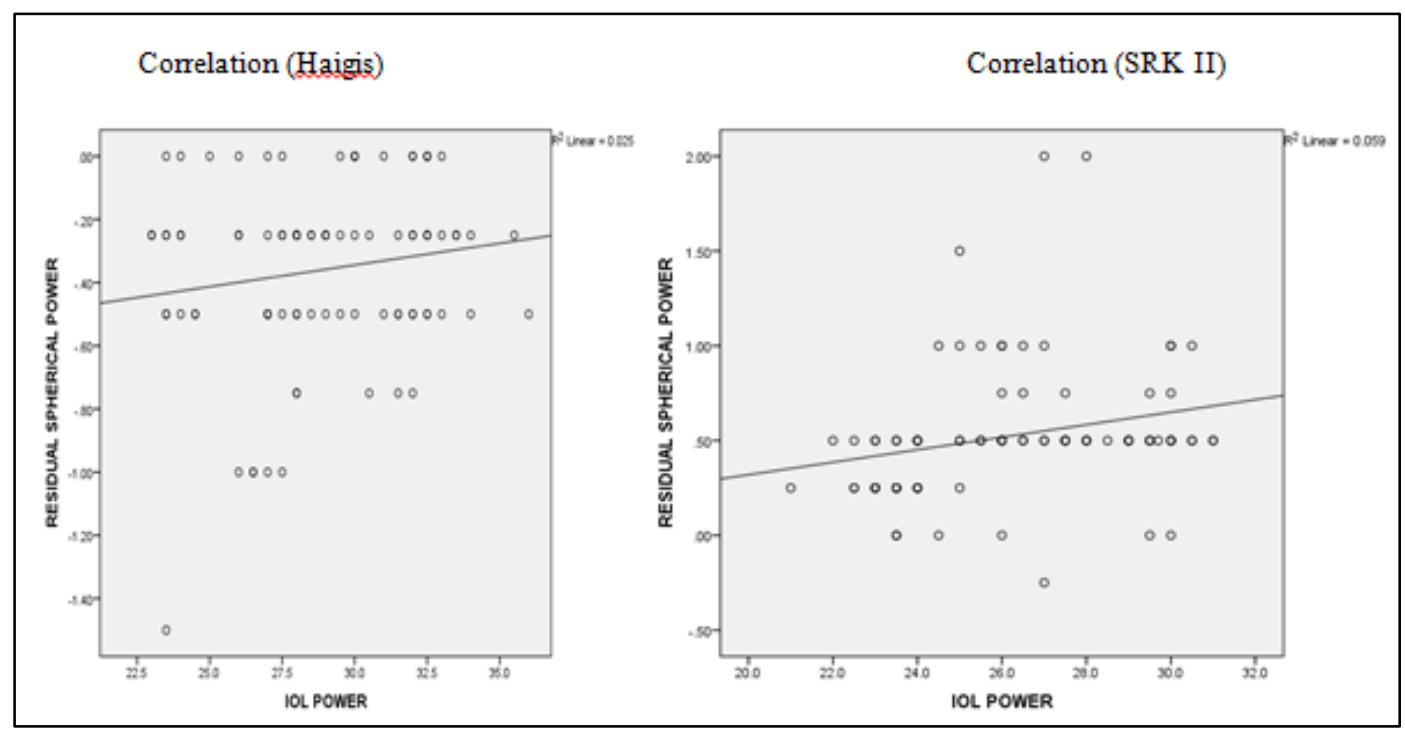

Fig. 4: Comparison of iol power with residual spherical power in Haigis and SRK II formulae.

On comparison of Haigis and SRK II formulae a negative correlation was found between axial length $(\mathrm{p}=0.047 *)$ with residual spherical power $\left(\mathrm{p}=0.009^{*}\right)$ which was statistically significant. A weak insignificant positive correlation was found with Haigis and a weak insignificant negative correlation was found with SRK II formulae when we compared the anterior chamber depth and the residual spherical power. A significant positive correlation with SRK II $\left(0.017^{*}\right)$ formulae and Haigis formulae was found when we compared the IOL power with the residual spherical power (Table 8, Fig. 2,3,4).

\section{Discussion}

Accurate biometry is central to obtaining emmetropia following cataract surgery. Errors in calculation of IOL power can result in residual spherical error. $1 \mathrm{~mm}$ error in axial length can induce a postoperative refractive error of 3 diopters. ${ }^{3}$ Newer formulae for IOL power calculation like Haigis formula aims to reduce this error by employing an additional preoperative specifics, the anterior chamber depth (ACD) ${ }^{6,8,9}$ This formula uses axial length and preoperative anterior chamber depth to estimate the postoperative effective lens position, thus reducing the errors in calculation of IOL power. ${ }^{3}$ Various studies have reported that Haigis formula gives a good refractive outcome in short eyes. ${ }^{6} 10-12$ On comparing eyes with short $\mathrm{AL},<22.0 \mathrm{~mm}$ Haigis and Hoffer Q formulae were found to be comparably accurate in eyes with ACD $>2.4 \mathrm{~mm}$, but Haigis formula was found to be more accurate than Hoffer Q formula in eyes with short $\mathrm{AL}$ when anterior chamber depth was less than $2.4 \mathrm{~mm}^{6}$

In our study, all our subjects were operated by the same surgeon and implanted with the same type of IOL and biometry was calculated by a single person to eliminate the differences due to difficulties in IOL constant optimization, disparities in visual outcome due to IOL design or material and to reduce interobserver variations. The visual outcome of patients at 6 weeks was considered to ensure postoperative refractive stability. ${ }^{13}$ All patients obtained a visual acuity of $6 / 24$ or more, but it was observed that the visual outcome of the group implanted with IOL calculated using Haigis formula was better than the group implanted with IOL calculated using SRK II formula. The number of participants who achieved best corrected visual acuity of $6 / 6$ and 6/9 were almost equal for both the groups which further points out that wrong IOL power calculation was the cause for the reduced visual acuity.

13 subjects did not achieve a BCVA of 6/6. The cause for lesser BCVA was analysed. We found that 3 patients had cystoid macular oedema, 4 had ARMD changes and the rest had RPE changes and pigmentation of the macula.

An error of $> \pm 0.25$ was taken as significant residual spherical error. 51 eyes achieved good postoperative visual outcome using Haigis formula while only 19 eyes achieved a good postoperative visual outcome using SRK II formula.

When SRK II formula was used a large number of subjects had a spherical error of $\pm 0.5 \mathrm{D}$ (50 eyes i.e., $57.5 \%)$. While most subjects had $< \pm 0.25 \mathrm{D}$ (51 eyes i.e., $58.6 \%$ ) spherical error when Haigis formula was used. All subjects implanted with IOL calculated with Haigis formula had a spherical error $\leq 1 \mathrm{D}$ while 3 patients had a residual spherical error more than 1D. Application of SRK II formula in eyes with short AL may lead to postoperative surprises. In our study 2 subjects had a residual spherical error of $+2 \mathrm{D}$. In this study we observed that using Haigis formula in eyes with short AL gave a better postoperative outcome.

The highest power calculated using Haigis formula was $36 \mathrm{D}$ and $31 \mathrm{D}$ with SRK II formula. Haigis formula when employed in eyes with short AL led to a mild over correction which is consistent with findings of other studies. ${ }^{14,15}$ Using SRK II formula led to a mild under correction.

We analysed the correlation between axial length and the anterior chamber depth and found that shorter eyes tend to have a shorter anterior chamber depth. 


\section{Conclusion}

The postoperative refraction of patients implanted with IOLs calculated using Haigis formula was closer to emmetropia. Haigis formula when used in eyes with short AL led to a mild over correction leading to a slightly more myopic outcome while SRK II formula led to a mild under correction leading to a mild hypermetropic outcome. A negative correlation was noted between axial length and residual spherical power and was statistically significant. A weak positive correlation was found with Haigis and a weak negative correlation was found with SRK II formulae when we compared the anterior chamber depth and the residual spherical power which was not statistically significant.

\section{Source of Funding: None.}

\section{Conflict of Interest: None.}

\section{References}

1. Brian G, Taylor H. Cataract blindness- challenges for the $21^{\text {st }}$ century. Bull World Health Organ. 2001;79:249-56.

2. Haigis W. The Haigis formula. In: Shammas HJ, ed. Intraocular Lens Power Calculations. Thorofare, NJ: Slack. 2004:41-57.

3. Garg A, Lin JT, Latkany R, Bovet J, Haigis W, editors. Mastering techniques of IOL power calculations. $2^{\text {nd }}$ ed. New Delhi: Jaypee brothers medical publishers (P) LTD;2009.

4. Ladas LJ, Siddiqui AA, Devgan U, Jun AS. A 3-D "super surface" combining modern intraocular lens formulas to generate a 'super formula' and maximize accuracy. JAMA Ophthalmol. 2015;133:1431-6.

5. Olsen T, Thim K, Corydon L. Accuracy of the newer generation intraocular lens power calculation formulas in long and short eyes. J Cataract Refract Surg. 1991;17:187-93.

6. Eom Y, Kang S, Song JS, Kim YY, Kim HM. Comparison of Hoffer Q and Haigis for intraocular lens power calculation according to anterior chamber depth in short eyes. Am J Ophthalmol. 2014;157:818-24.
7. Jeong J, Song H, Lee J K, Chuck RS, Kwon J. The effect of ocular biometric factors on the accuracy of various IOL power calculation formulas. BMC Ophthalmol. 2017;17:62.

8. Lee AC, Qazi MA, Pepose JS. Biometry and intraocular lens power calculation. Curr Opin Ophthalmol. 2008;19:13-7.

9. Bang S, Edell E, Yu Q, Pratzer K, Stark W. Accuracy of intraocular lenscalculations using the IOLMaster in eyes with long axial length and acomparison of various formulas. Ophthalmol. 2011;118:503-6.

10. Moschos MM, Chatziralli IP, Koutsandrea C. Intraocular lens power calculation in eyes with short axial length. Indian $J$ Ophthalmol. 2017;62:692-4.

11. Carifi G, Aiello F, Zygoura V, Kopsachilis N, Maurino V. Accuracy of refractive prediction determined by multiple currently available intraocular lens power calculation formulas in small eyes. Am J Ophthalmol. 2015;159:577-83.

12. Roh YR, Lee SM, Han YK, Kim MK, Wee WR, Lee JH et al. Intraocular lens power calculation using IOL master and various formulas in short eyes. Korean J Ophthalmol. 2011;25:151-5.

13. Hoffer KJ, Aramberri J, Haigis W, Olsen T, Savini G, Shammas HJ, et al. Protocols for studies of intraocular lens formula accuracy. Am J Ophthalmol. 2015;160:403-5.

14. MacLaren RE, Natkunarajah M, Riaz Y, Bourne RRA, Restori $\mathrm{M}$, Allan BDS et al. Biometry and formula accuracy with intraocular lenses used for cataractsurgery in extreme hyperopia. Am J Ophthalmol. 2007;143:920-31.

15. Terzi E, Wang L, Kohnen T. Accuracy of modern intraocular lens power calculation formulas in refractive lens exchange for high myopia and high hyperopia. J Cataract Refract Surg. 2009;35:1181-9.

How to cite this article: Kumar $M \mathrm{H}$, Sangeetha $\mathrm{T}$, Kanthamani, Babu NJ. Comparison of accuracy of haigis and SRK II formula for intraocular lens power calculation in eyes with short axial length. Indian J Clin Exp Ophthalmol. 2019;5(3):376-81. 